\title{
Room-Temperature Scanning Hall Probe Microscope (RT-SHPM) Imaging of Garnet Films Using New High-Performance InSb Sensors
}

\author{
A. Oral, M. Kaval, M. Dede, H. Masuda, A. Okamoto, I. Shibasaki, and A. Sandhu
}

\begin{abstract}
High-performance InSb micro-Hall sensors were fabricated by optical lithography and incorporated in a room-temperature scanning Hall probe microscope for imaging of localized magnetic fluctuations in close proximity to the surfaces of crystalline uniaxial garnet films. The room-temperature noise figure of the InSb sensors was 6-10 $\mathrm{mG} / \sqrt{ } \mathrm{Hz}$, which is an order of magnitude better than GaAs-AlGaAs two-dimensional electron gas sensors used to date.
\end{abstract}

Index Terms-Hall effect, scanning Hall probe microscopy.

\section{INTRODUCTION}

$\mathbf{S}$ CANNING Hall probe microscopy (SHPM) [1] is a quantitative and noninvasive technique for imaging localized surface magnetic field fluctuations such as ferromagnetic domains with high spatial and magnetic field resolution of $\sim 120 \mathrm{~nm}$ and $70 \mathrm{mG} / \sqrt{\mathrm{Hz}}$, respectively, at room temperature. This new technique offers advantages and complements the other magnetic imaging methods like magnetic force microscopy (MFM) [2], magnetic near field scanning optical microscopy[3], and Kerr microscopy [4]. In SHPM, a submicrometer Hall probe is scanned over the sample surface to measure the perpendicular component of the surface magnetic fields using conventional scanning tunneling microscopy (STM) positioning techniques as shown in Fig. 1. The SHPM system can be designed to enable operation over a wide temperature range $(4 \mathrm{~K}-300 \mathrm{~K})$, and we have previously reported on the excellent properties of GaAs-GaAlAs two-dimensional electron gas (2DEG) Hall probes for cryogenic and room-temperature measurements [5], [6]. In an attempt to overcome Hall sensor drive current limitations due to carrier depletion effects in submicrometer GaAs-AlGaAs 2DEG probes at room temperature, we recently fabricated bismuth (Bi) nano-Hall sensors using focused ion beam milling and achieved a spatial resolution of $120 \mathrm{~nm}$ [7]. However, the minimum detectable

Manuscript received February 15, 2002; revised May 28, 2002. This work was supported in part by TÜBİTAK under Grant TBAG-1878, by Bilkent University under the Faculty Development Grant and Research Grant, by NanoMagnetics Instruments Ltd. U.K. under Grant NM1-01, and by the Japanese Ministry of Education, Culture, Sports, Science and Technology under Grant 13650354.

A. Oral, M. Kaval, and M. Dede are with the Department of Physics, Bilkent University, 06533 Ankara, Turkey (e-mail: ahmet@fen.bilkent.edu.tr).

H. Masuda is with the Toei Kogyo Ltd., Machida 1940035, Japan.

A. Okamoto and I. Shibasaki are with the Asahikasei Corporation, Fuji City 416-8501, Japan.

A. Sandhu is with the Department of Electrical and Electronic Engineering, Tokai University, Hiratsuka 259-1292, Japan (e-mail: sandhu@keyaki.cc.utokai.ac.jp).

Digital Object Identifier 10.1109/TMAG.2002.803607.

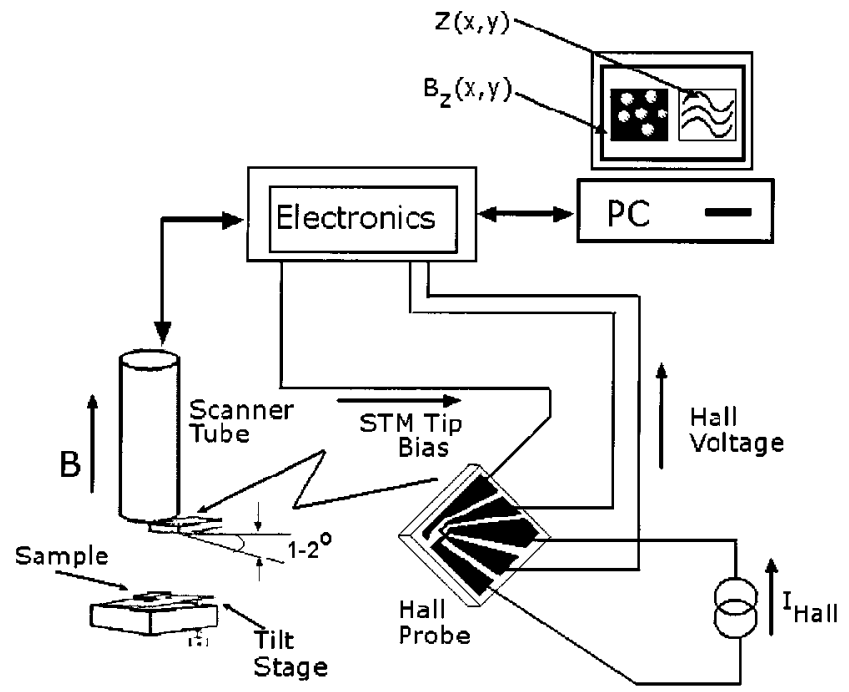

Fig. 1. Schematic diagram of RT-SHPM.

magnetic field with the Bi nano-Hall sensors was high due to high carrier concentration and low mobility at the room temperature. A promising alternative material for fabricating submicrometer Hall sensors is InSb, which has both of the desired properties (i.e., low carrier concentration and high mobility) at room temperature compared to GaAs-AlGaAs 2DEG and $\mathrm{Bi}$ thin films. In this paper, we report on the development of new low-noise, high-performance InSb thin film micro-Hall sensors for room-temperature scanning Hall probe microscopy (RT-SHPM) exhibiting a noise level of $6-10 \mathrm{mG} / \sqrt{ } \mathrm{Hz}$, which is an order of magnitude better than GaAs-AlGaAs $2 \mathrm{DEG}$ sensors.

\section{EXPERIMENT}

\section{A. Scanning Hall Probe Microscope}

The RT-SHPM [8] used in this study has a scan range of $50 \times 50 \mu \mathrm{m}$ in $X Y$ directions and $4.8 \mu \mathrm{m}$ in $Z$ direction. The SHPM incorporates $X Y Z$ motors for coarse micropositioning, a video camera for Hall sensor alignment, and an integrated coil concentric to the Hall sensor head for the application of external magnetic fields of up to \pm 40 Oe. Furthermore, a newly developed compact sized powerful pulse coil can be coupled with the system to apply external fields up to \pm 25000 Oe. The Hall sensor is positioned close to a gold-coated corner of a deep etch mesa, which serves as STM tip. The Hall probe chip is tilted $\sim 1.25^{\circ}$ with respect 


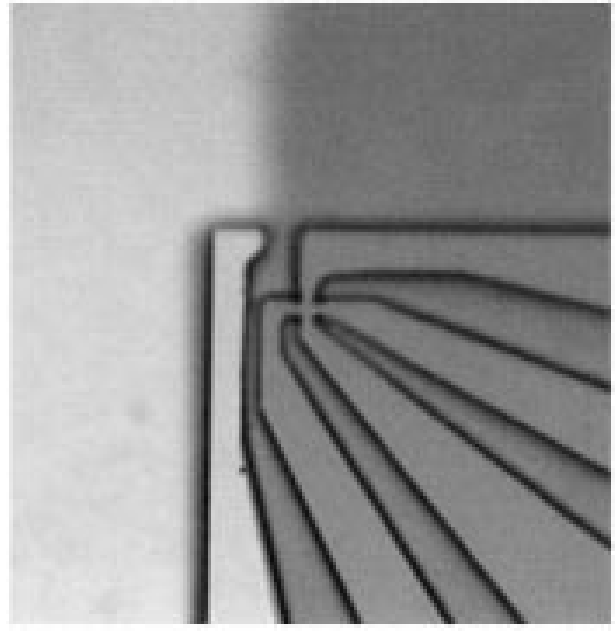

Fig. 2. Optical micrograph of a finished InSb thin film Hall probe. Gold-coated corner of the chip on the left of Hall cross serves as STM tip.

to sample ensuring that the corner of the mesa is the highest point. The microscope can be run in two modes: STM tracking and liftoff mode. In the STM tracking mode, the tunnel current between the corner of the Hall sensor chip and the sample is measured and used to drive the feedback loop enabling the simultaneous measurement of both STM topography and the magnetic field distribution of the sample surface. This mode of operation gives the highest sensitivity because of the smallest probe-sample separation but with the drawback of being slow. In the liftoff mode, the Hall sensor is lifted off to a certain height above the sample and the head can be scanned extremely fast ( $\sim 4 \mathrm{~s} /$ frame) for measurements of the local magnetic field distribution.

\section{B. InSb Hall Probe Microfabrication}

The InSb micro-Hall probes were fabricated using high-quality epitaxial InSb thin films with a thickness of $1 \mu \mathrm{m}$ grown by MBE on semi-insulating GaAs substrate [9], [10]. Standard room-temperature van der Pauw measurements showed the InSb films to have a carrier concentration of $2 \times 10^{12} \mathrm{~cm}^{-2}$ and a Hall mobility of $55500 \mathrm{~cm}^{2} / \mathrm{Vs}$. The process used for the microfabrication of the InSb Hall sensors was similar to the GaAs 2DEG sensors as reported previously [1]. The differences on this occasion were: 1) reactive ion etching was used for Hall probe definition and 2) Ti-Au Ohmic contacts. Fig. 2 shows a typical $\sim 1.5 \mu \mathrm{m}$ $\times 1.5 \mu \mathrm{m} \mathrm{InSb}$ micro-Hall probe with a Hall coefficient of $R_{H} \sim 0.034 \Omega /$ Gauss and a series resistance of $R_{s}=2.2 \mathrm{k} \Omega$.

\section{RESULTS}

\section{A. Hall Probe Noise Measurements}

The InSb micro-Hall sensors were driven with a dc current $\left(I_{\mathrm{HALL}}\right)$ and the Hall voltage measured using a low-noise amplifier positioned close to the Hall probe. The amplifier's gain and bandwidth were an adjustable parameter. The minimum detectable magnetic field with a Hall probe can be written as [1]

$$
B_{\min }=V_{\text {noise }} /\left(R_{H} I_{\mathrm{HALL}}\right)
$$

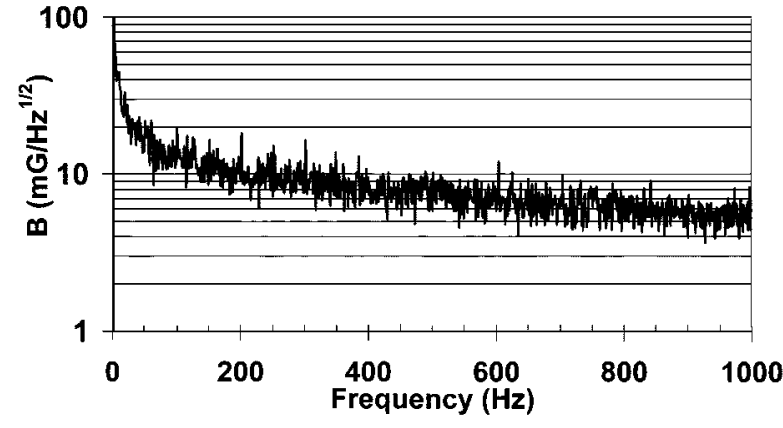

(a)

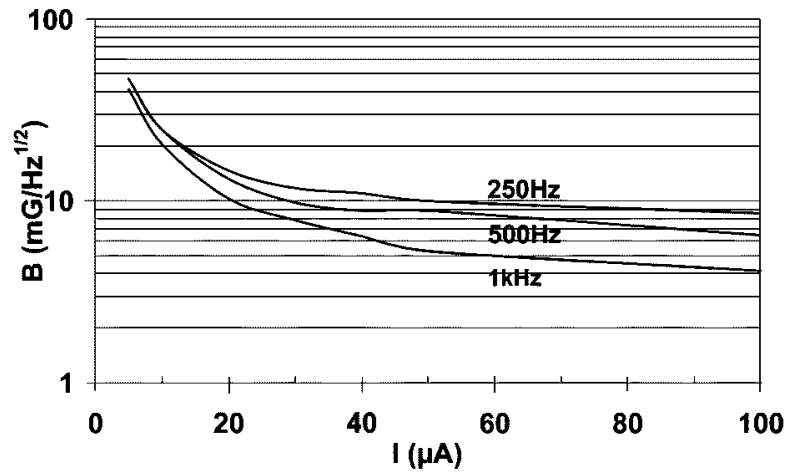

(b)

Fig. 3. (a) Noise spectrum of InSb Hall probe at $I_{\text {HALL }}=50 \mu \mathrm{A}$. (b) Magnetic field noise of InSb Hall probe as a function of Hall current.

where $V_{\text {noise }}$ is the total voltage noise at the input of the Hall amplifier. The $V_{\text {noise }}$ has two components; voltage noise of the amplifier and the noise due to the series resistance of the Hall sensor $\left(R_{s}\right)$. It is desirable to drive the Hall probe with the highest permissible current. However, the voltage noise of series resistance $R_{S}$ increases due to heating of the charge carriers and the lattice. Therefore, the Hall current cannot be increased indefinitely and there is a maximum useable $I_{\text {HALL max }}$.

We measured the noise spectra of the InSb micro-Hall sensors at different Hall currents to find optimum operating conditions and $I_{\text {HALL max }}$. The gain and bandwidth of the Hall amplifier were set to 10000 and $1 \mathrm{kHz}$, respectively. The noise spectra were measured using a fast Fourier transform signal analyzer in a $1 \mathrm{~Hz}$ equivalent bandwidth at different Hall currents. Fig. 3(a) shows the noise spectrum for $I_{\mathrm{HALL}}=50 \mu \mathrm{A}$. Minimum detectable magnetic field is plotted as a function of Hall current in Fig. 3(b) at three different measurement frequencies; 250, 500, and $1000 \mathrm{~Hz}$. The noise decreases slightly at higher frequencies. This could be due to spurious capacitances between the measurement leads of the Hall probe, which reduces the bandwidth of the Hall voltage signal. The minimum detectable magnetic field can be conservatively estimated to be $6-10 \mathrm{mG} / \sqrt{ } \mathrm{Hz}$ at $I_{\text {HALL }}=50 \mu \mathrm{A}$. The new InSb sensors are compared with the Hall probes microfabricated from a GaAs 2DEG material. Fig. 4(a) shows the noise spectrum of the $1.5 \mu \mathrm{m}$ GaAs Hall sensor with a Hall coefficient of $R_{H} \sim 0.26 \Omega /$ Gauss and a series resistance of $R_{s}=105 \mathrm{k} \Omega$ at $I_{\mathrm{HALL}}=2.5 \mu \mathrm{A}$. Minimum detectable magnetic field is plotted as a function of Hall current in Fig. 4(b) at three different measurement frequencies; 250, 500, and $1000 \mathrm{~Hz}$. The noise levels change from sensor to 


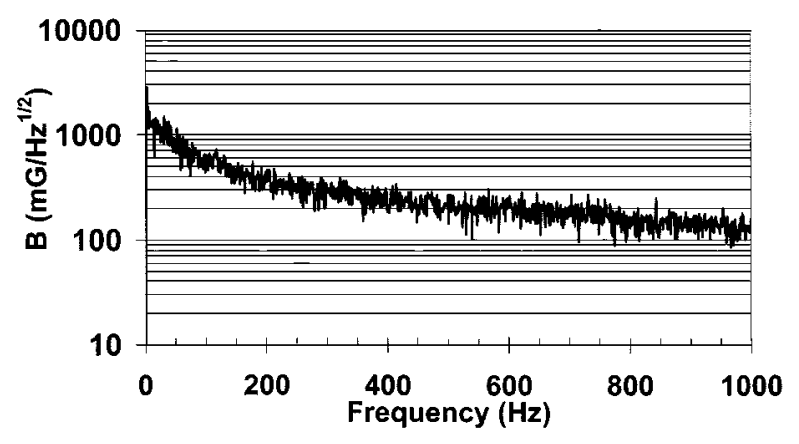

(a)

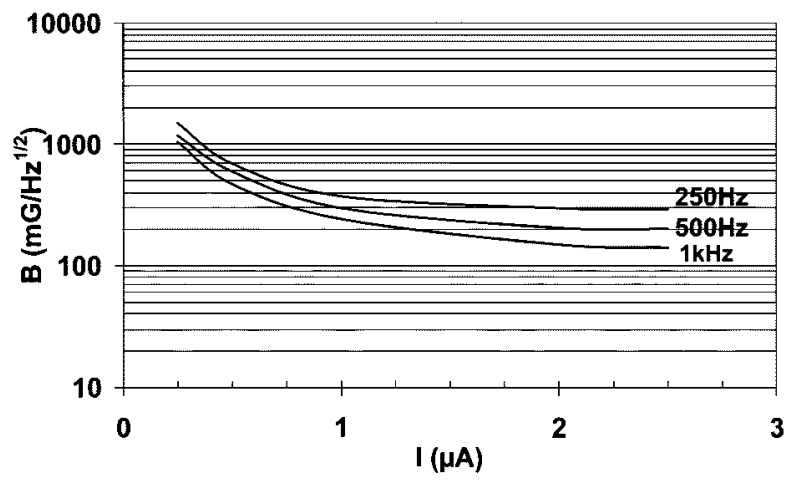

(b)

Fig. 4. (a) Noise spectrum of GaAs $2 \mathrm{DEG}$ Hall probe at $I_{\mathrm{HALL}}=2.5 \mu \mathrm{A}$. (b) Magnetic field noise of GaAs 2DEG Hall probe as a function of Hall current.

sensor and the data shown in the Fig. 4 is not from the best GaAs 2DEG Hall sensor.

\section{B. Imaging Garnet Crystal With InSb Micro-Hall Probe}

The newly developed InSb micro-Hall sensors showed approximately ten times better noise performance compared to conventional GaAs-based sensors used in RT-SHPM. These new InSb thin-film Hall probes were used to image magnetic domain structures of crystalline garnet films which we have studied previously using GaAs 2DEG Hall sensors [11]. The sample is vacuum coated with a thin 20 -nm layer of gold film to enable SHPM imaging. Fig. 5(a)-(c) shows $25 \mu \mathrm{m} \times 25 \mu \mathrm{m}$ RT-SHPM images of 5.5- $\mu \mathrm{m}$-thick crystalline Bi-substituted iron garnet films measured using Hall currents of 3, 30, and $50 \mu \mathrm{A}$, respectively. The images are acquired in the liftoff mode with the InSb micro-Hall sensor at a height of $0.35 \mu \mathrm{m}$. The black and white regions correspond to surface field fluctuations into and out of the plane of the paper ranging between $\pm 52 \mathrm{G}$. We did not observe any differences in the images obtained using Hall currents greater than $50 \mu \mathrm{A}$.

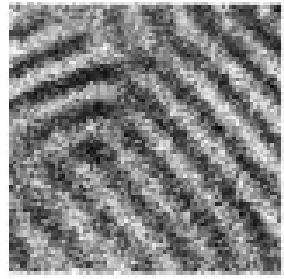

(a)

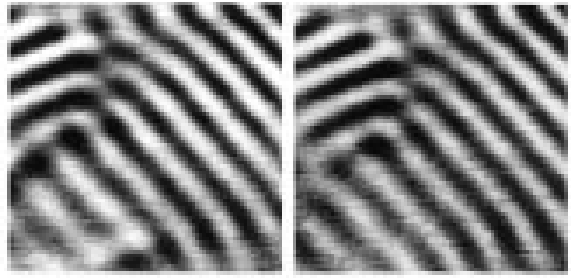

(b) (c)
Fig. 5. Typical $25 \mu \mathrm{m} \times 25 \mu \mathrm{m}$ RT-SHPM images of 5.5- $\mu \mathrm{m}$-thick crystalline bismuth-substituted iron garnet films obtained with Hall currents of (a) 3, (b) 30, and (c) $50 \mu \mathrm{A}$

\section{CONCLUSION}

InSb micro-Hall probes were shown to be highly promising, low-noise alternatives to GaAs sensors for RT-SHPM with an order of magnitude better performance. Further improvements in the spatial resolution $(\sim 50 \mathrm{~nm})$ and magnetic sensitivity of room-temperature InSb Hall probes can be envisaged by using thinner InSb films, InSb quantum wells, and InSb 2DEG structures.

\section{REFERENCES}

[1] A. Oral, S. J. Bending, and M. Henini, "Real-time scanning Hall probe microscopy," Appl. Phys. Lett., vol. 69, pp. 1324-1326, Aug. 1996.

[2] Y. Martin and H. K. Wickramasinghe, "Magnetic imaging by force microscopy with $1000 \AA$ resolution," Appl. Phys. Lett., vol. 50, pp. $1455-1457$, May 1987.

[3] E. Betzig, J. K. Trautman, R. Wolfe, E. M. Gyorgy, P. L. Finn, M. H. Kryder, and C. H. Chang, "Near-field magnetooptics and high-density data-storage," Appl. Phys. Lett., vol. 61, pp. 142-144, July 1992.

[4] F. Schmidt and A. Hubert, "Domain observations on CoCr-layers with a digitally enhanced Kerr-microscope," J. Magn. Magn. Mater., vol. 61, pp. 307-320, Oct. 1986.

[5] A. Oral, S. J. Bending, and M. Henini, "Scanning Hall probe microscopy of superconductors and magnetic materials," J. Vac. Sci. Technol. B., vol. 14, pp. 1202-1205, Mar-Apr. 1996.

[6] A. Sandhu, H. Masuda, A. Oral, and S. J. Bending, "Direct magnetic imaging of ferromagnetic domain structures by room temperature scanning Hall probe microscopy using a bismuth micro-Hall probe," Jpn. J. Appl. Phys., pt. 2, vol. 40, pp. L524-L527, May 2001.

[7] A. Sandhu, H. Masuda, K. Kurosawa, A. Oral, and S. J. Bending, "Bismuth nano-Hall probes fabricated by focused ion beam milling for direct magnetic imaging by room temperature scanning Hall probe microscopy," Electron. Lett., vol. 37, pp. 1335-1336, Oct. 2001.

[8] Room Temperature Scanning Hall Probe Microscope (RT-SHPM). NanoMagnetics Instruments Ltd., 17 Croft Road, Oxford, U.K. [Online]. Available: www.nanomagnetics-inst.com

[9] A. Okamoto, A. Ashihara, T. Akaogi, and I. Shibasaki, "Thin films grown on GaAs substrate and their magneto-resistance effect," J. Cryst. Growth, vol. 227-228, pp. 619-624, July 2001.

[10] I. Shibasaki, A. Okamoto, A. Ashihara, and K. Suzuki, "Properties and applications of InSb single crystal thin film Hall elements," in Tech. Dig. 18th Sensor Symp., 2001, pp. 233-238.

[11] A. Sandhu, H. Masuda, A. Oral, and S. J. Bending, "Room temperature sub-micron magnetic imaging by scanning Hall probe microscopy," Jpn. J. Appl. Phys., pt. 1, vol. 40, pp. 4321-4324, June 2001. 